\title{
Implant Insertion after Using of Oxidized Regenerated Cellulose as a Graft Material in a Transalveolar Maxillary Sinus Floor Elevation
}

\author{
Ahmed AH El-Feky* \\ Assistant Professor, Department of Oral and Maxillofacial Surgery, Faculty of Dental Medicine for Boys, \\ Al-Azhar University, Cairo, Egypt
}

*Corresponding author: Ahmed AH El-Feky, Assistant Professor, Department of Oral and Maxillofacial Surgery, Faculty of Dental Medicine for Boys, Al-Azhar University, Cairo, Egypt

\begin{abstract}
Objectives: To evaluate the use of oxidized regenerated cellulose as a graft material in trans alveolar maxillary sinus floor elevation and implant insertion.

Patient and methods: A clinical study was conducted on sixteen patients, suffering from partially edentulous areas at the maxillary posterior region. Sixteen patients with 18 implants were divided randomly into two equal groups 8 patients with 9 implants in each group.

Study group, patients were subjected to trans alveolar sinus lift and Oxidized cellulose (SurgicelSNoW) ${ }^{\circledR}$ Absorbable Hemostat, Ethicon Inc., USA) graft were applied and immediate implant placement.

Control group, patients were subjected to trans alveolar sinus lift and implants applied without any graft. All patients were followed up clinically and radiographically by Cone Beam Computed Tomography (CBCT) at the intervals of 6 and 9 months to evaluate bone density and bone height around dental implants and stability of the dental implants.

Results: There was no statistically significant difference between the two groups regarding the mean of change in IQS values between the immediate and 6 months postoperatively, while there was highly statistically significant increase in the ISQ in both groups, the radiographic parameters of this study include measuring bone height and density of neoformed bone, where there was a highly significant increase in the height in a study group (performed by ORC graft) more than the control group (performed without any graft). Both techniques resulted in successful clinically stable implants after a follow-up period of up to 9 months.
\end{abstract}

Conclusion: According to the results of this study we could conclude that: Osteotome mediated maxillary sinus lifting with simultaneous implant placement using oxidized regenerated cellulose graft is a promising technique compared to other grafting materials as technically safe, cheap, fast and its application does not require a sophisticated procedure through trans alveolar sinus membrane elevation and simultaneous implant insertion. Oxidized regenerated cellulose could be considered as an alternative reasonable grafting material with comparable outcomes to osteon II with less postoperative complications, if the residual bone below sinus floor $\geq 8 \mathrm{~mm}$, it's not required to use graft materials for sinus floor augmentation and if the residual bone height is $\leq$ $6 \mathrm{~mm}$ ORC could be used as filling material for crestal sinus floor augmentation.

\section{Keywords}

Maxillary sinus lift, Oxidized regenerated cellulose (ORC), Dental implants

\section{Introduction}

The edentulous ridge in the posterior maxilla is characterized by progressive and irreversible vertical bone resorption after tooth extraction, this leads to an atrophic bone situation and limits the application of implant therapy. In such cases, the sinus lift procedure is indicated; it is expected to provide sufficient bone for optimal implant osseointegration, and provide longterm success [1].

The lateral window technique is the most frequently used procedure for vertical bone augmentation of the atrophic posterior maxilla. An alternative to the lateral approach is the osteotome sinus floor elevation

Citation: El-Feky AAH (2021) Implant Insertion after Using of Oxidized Regenerated Cellulose as a Graft Material in a Transalveolar Maxillary Sinus Floor Elevation. Res Rep Oral Maxillofac Surg 5:051. doi.org/10.23937/2643-3907/1710051

Accepted: June 21, 2021, 2020; Published: June 23, 2021

Copyright: (C) 2021 El-Feky AAH. This is an open-access article distributed under the terms of the Creative Commons Attribution License, which permits unrestricted use, distribution, and reproduction in any medium, provided the original author and source are credited. 
procedure. It is less invasive and the treatment can be achieved with a single surgery [2]. Another use of short implants with a textured surface in sites with limited residual bone height, the surgical procedure is simpler, and the treatment duration can be reduced [3]. The latter was even found to be inversely correlated with the residual bone height [4]. To enable placement of implants other than short one because the minimum length for predictable dental implant success is $10 \mathrm{~mm}$ or what is called standard implant length.

Recently, the need for autologous bone grafts and grafting material to achieve successful sinus augmentation procedures have been questioned $[5,6]$. Researches has not reached an agreement about the most suitable material for sinus augmentation $[7,8]$. Some authors used oxidized regenerated cellulose (ORC), which is a hemostatic agent as a graft biomaterial with a superiority to the autologous bone in terms of new bone formation to serve as an osteoconductive material and scaffold for new bone formation.

Gray, et al. evaluated the efficacy of oxidized regenerated cellulose in sinus lift by $\mathrm{MRI}$, the result showing similarity between the graft and normal bone results to that of the bone.

\section{Patient and Method}

\section{Study design and population}

The study included (sixteen) patients. All patients suffered from partially edentulous areas with deficient ridge height $\leq 6 \mathrm{~mm}$ indicated for transcrestal osteotome sinus augmentation and simultaneous dental implant place-mentat maxillary posterior region. The patients were selected from the outpatient clinic of Oral and Maxillofacial Surgery Department, Faculty of Dental Medicine, Boys, Cairo Al-Azhar University. Selection of patient was done according to inclusion criteria, good healthy patients, free from a systemic disease such as diabetes mellitus, patient age ranged from 20 to 55 years of both genders, patients with edentulous posterior maxilla andadequate subantral bone height $\geq$ $5 \mathrm{~mm}$ to ensure primary stability for the placement of implants (single-stage surgery). The exclusion criteria included, immunocompromized patients, presence of active infection or inflammation at the time of operation, patients with previous history of radio and/or chemotherapy treatment in the head and neck region, patients with previous history of oral or intravenous bisphosphonates and unmanaged psychiatric patients.

\section{Pre-surgical preparation}

- Clinical assessment of patient's past medical history, oral condition, evaluation by inspection and palpation of muscles of mastication, temporomandibular joint, and lymph nodes to check for any disorders. Big digital palpation to assess any sharp bony projections, undercuts, or soft tissue swellings.

- Radiographic evaluation including preoperative panoramic radiographs: The maxillary sinus was examined to detect any pathology that interfered with sinus lifting. Cone Beam CT (CBCT): Were taken for every patient to determine alveolar bone height beneath the sinus floor and alveolar bone width which were indicated for transalveolar sinus lift technique.

\section{Surgical procedure}

All patients were operated on under local anesthesia (using Articaine hydrochloride $4 \%$ with epinephrine 1:100 000), Paracrestal incision with palatal bias was done, the full-thickness mucoperiosteal flap was reflected, sequential drilling was done in a vertical direction and moved up and down during drilling with light intermittent finger pressure to permit continuous internal and external coolant accomplished first with pilot drill to the predetermined height (1-2 mm less than vertical height between sinus floor and alveolar ridge crest). Sinus lifting were performed in the same manner in both groups by greenstick fracture of the sinus floor with osteotome type A by gentle firm tapping while asking the assistant to support the patient's head. After examining the integrity of sinus membrane by Valsalva maneuver; digital periapical radiograph was taken to assess the sinus elevation made using osteotome. In the study group after sinus lifting, aspirated fresh blood collected in tubes and ORC strips saturated with it. Saturated ORC strips inserted in the osteotomy site and pressed by osteotome against the broken sinus floor elevate it. After the osteotomy site was completely performed and ORC graft inserted the implant was handled to its position inside the osteotomy site. After complete insertion of an implant into the bone the cover screw was tightened, suturing of the flap was done with 3-0 black silk suture. While, in control group surgery procedure, flap design, drilling for implant and sinus lifting was done in the same manner as in the study group but the implant was inserted without any graft, until its top flushed with the bone surface. The cover screw was tightened and suturing of the flap was done with 3-0 black silk suture Figure 1.

The implant stability was realized by the residual alveolar bone, at more than 3 to $4 \mathrm{~mm}$ with underosteotomy procedure simultaneous implant placement can be made successfully, if implants are directed palatal to engage nasopalatine bone, higher stability is achieved. When tenting of the Schneiderian membrane achieved and clot stabilized, bone formation occurs similar like extraction sockets.

\section{Post-surgical care}

Postoperative antibiotics and analgesics were prescribed. Patients were instructed for maintaining 
Table 1: Comparison between the two studied groups according to bone height.

\begin{tabular}{|l|l|l|l|l|}
\hline & $\begin{array}{l}\text { Study } \\
(\mathbf{n = 8})\end{array}$ & $\begin{array}{l}\text { Control } \\
(\mathbf{n}=\mathbf{8})\end{array}$ & Test of sig. & P \\
\hline Bone Height & & & & \\
\hline Pre & $5.93 \pm 0.79$ & $8.60 \pm 0.21$ & $\mathrm{t}=9.238^{*}$ & $<0.001^{*}$ \\
\hline Post & $11.13 \pm 1.36$ & $11.0 \pm 0.0$ & $\mathrm{t}=0.261$ & 0.802 \\
\hline \% Change & $88.22 \pm 8.77$ & $27.98 \pm 3.18$ & $\mathrm{U}=0.0^{*}$ & $<0.001^{*}$ \\
\hline
\end{tabular}

t: Student t-test; U: Mann Whitney test; P: P-value for comparing between the studied groups; *: Statistically significant at P $\leq 0.05$

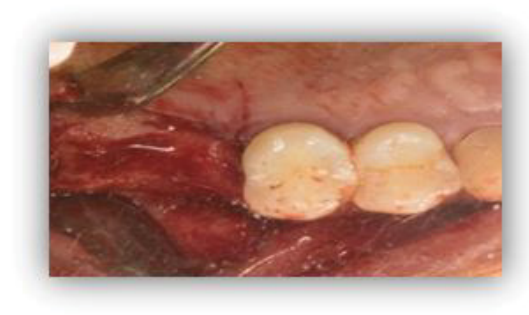

A

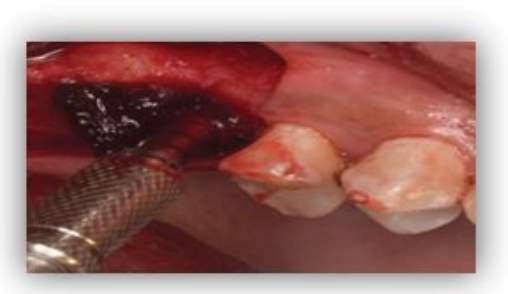

B

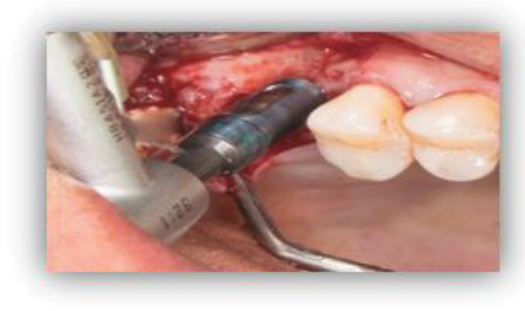

C

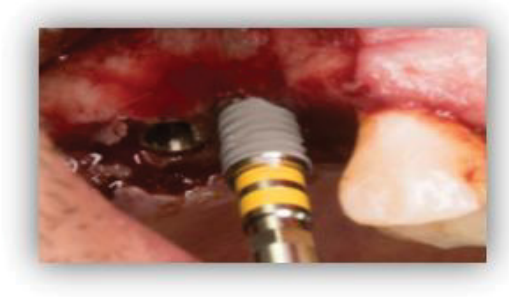

D

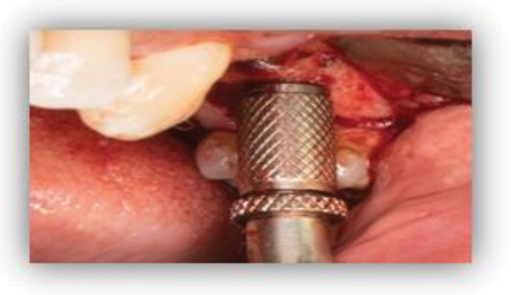

$\mathrm{E}$

Figure 1: Photograph of implant insertion showing A) Alveolar ridge after flap reflection in both groups; B) Implant drilling osteotomy in both groups; C) ORC graft pressed by osteotome against sinus floor in the study group; D) Sins floor elevation with osteotome without graft in control group; E) Implant insertion inside the osteotomy site.

good oral hygiene with Chlorhexidine $\mathrm{HCL}(0.12 \%)$. All patients were instructed to bite on the pack for 30 min and have a soft diet for the first week. For those having bilateral implants; a soft diet was maintained for 3 weeks.

\section{Post-operative assessment}

Suture removal was performed after 8-10 days. At 6 months, the clinical and radiographic evaluation was done to all cases, as the following:

Clinical evaluation: All patients were examined to check for the presence of pain recorded in the Visual Analog Scale (VAS of 10), discomfort, swelling. Osstell was used to assess implant stability and osseointegration, immediate and 6 months postoperatively.

Postoperative radiographic evaluation: Cone-beam was done preoperatively, immediately and at 6 months postoperatively, to assess bone density and bone height around dental implants. Measuring was done with Cone- beam computed tomography $\mathrm{CBCT}^{*}$ was performed 6 months after implantation for measuring the bone height of the ridges at mesial and distal points of the implant and for measuring of bone density around the apex of the implant.

Statistical analysis: The collected data were coded, processed, and analyzed using the SPSS ${ }^{*}$. Quantitative data were expressed as mean \pm SD (Standard deviation) and median (range). Independent samples t-test was used to compare between two independent groups of normally distributed variables (parametric data) while Mann Whitney $U$ test was used for non-normally distributed Data (non-parametric data).

For comparison of data at two different time points, paired-samples t-test was used to compare between two related groups of normally distributed variables (parametric data) while Wilcoxon Signed-Rank test was used for non-normally distributed Data (non-parametric data). 


\section{Results}

\section{Demographic data}

This study was conducted 16 patients, 8 patients ranged in age between 40.0-50.0 years with a mean age of $45.0 \pm 5.35$ years for the study group and 8 patients ranged in age between 41.0-48.0 years with a mean age $44.75 \pm 2.76$ years for the control group. The study group had 5 males and 3 females, while the control group had 4 males and 4 females. The effect of age and sex on healing was noticed but without a statistically significant difference $(p=0.619)$. Changes between groups were detected without statistically significant differences where $P(0.909)$.

\section{CBCT postoperative outcomes}

Bone height and density was evaluated in all groups with (CBCT). Bone height in the study group, the mean value of the Bone Height was $5.93 \pm 0.79$ pre-treatment and, $11.13 \pm 1.36$ post-treatment. The Bone Height was increased with a significant statistical difference, where $\left(P \ll 0.001^{*}\right)$. In the control group, the mean value of the bone height was $8.60 \pm 0.21$ pre-treatment and, $11.0 \pm 0.0$ post-treatment. The bone height in the study group was more than control group with a significant statistical difference, where $\left(P<0.001^{*}\right)$ Table 1 and Figure 2.

The density was evaluated in all groups with CBCT. In the Study group, the mean value of the density was $408.1 \pm 154.1$ pre-treatment, $408.4 \pm 149.0$ Immediate and, $456.4 \pm 144.7$ post-treatment. The density was increased with a significant statistical difference, where $\left(P<0.002^{*}\right)$. In the control group, the mean value of the density was $479.0 \pm 9.62$ pre-treatment, $490.0 \pm$ 37.42 Immediate and, $524.5 \pm 27.26$ post-treatment. The density was increased with a significant statistical

Table 2: Comparison between the two studied groups according to density.

\begin{tabular}{|l|l|l|l|l|}
\hline & $\begin{array}{l}\text { Study } \\
(\mathbf{n = 8})\end{array}$ & $\begin{array}{l}\text { Control } \\
(\mathbf{n = 8})\end{array}$ & U & P \\
\hline Density & & & & \\
\hline Pre & $408.1 \pm 154.1$ & $479.0 \pm 9.62$ & 16.0 & 0.105 \\
\hline Immediate & $408.4 \pm 149.0$ & $490.0 \pm 37.42$ & 16.0 & 0.105 \\
\hline Post & $456.4 \pm 144.7$ & $524.5 \pm 27.26$ & 24.0 & 0.442 \\
\hline \% Change from pre to & & & & \\
\hline Immediate & $\uparrow 0.81 \pm 5.53$ & $\uparrow 2.20 \pm 5.76$ & 32.0 & 1.000 \\
\hline Post & $\uparrow 14.06 \pm 7.62$ & $\uparrow 9.44 \pm 3.49$ & 24.0 & 0.442 \\
\hline
\end{tabular}

$\mathrm{U}$ : Mann Whitney test; $\mathrm{p}$ : p-value for comparing between the studied groups

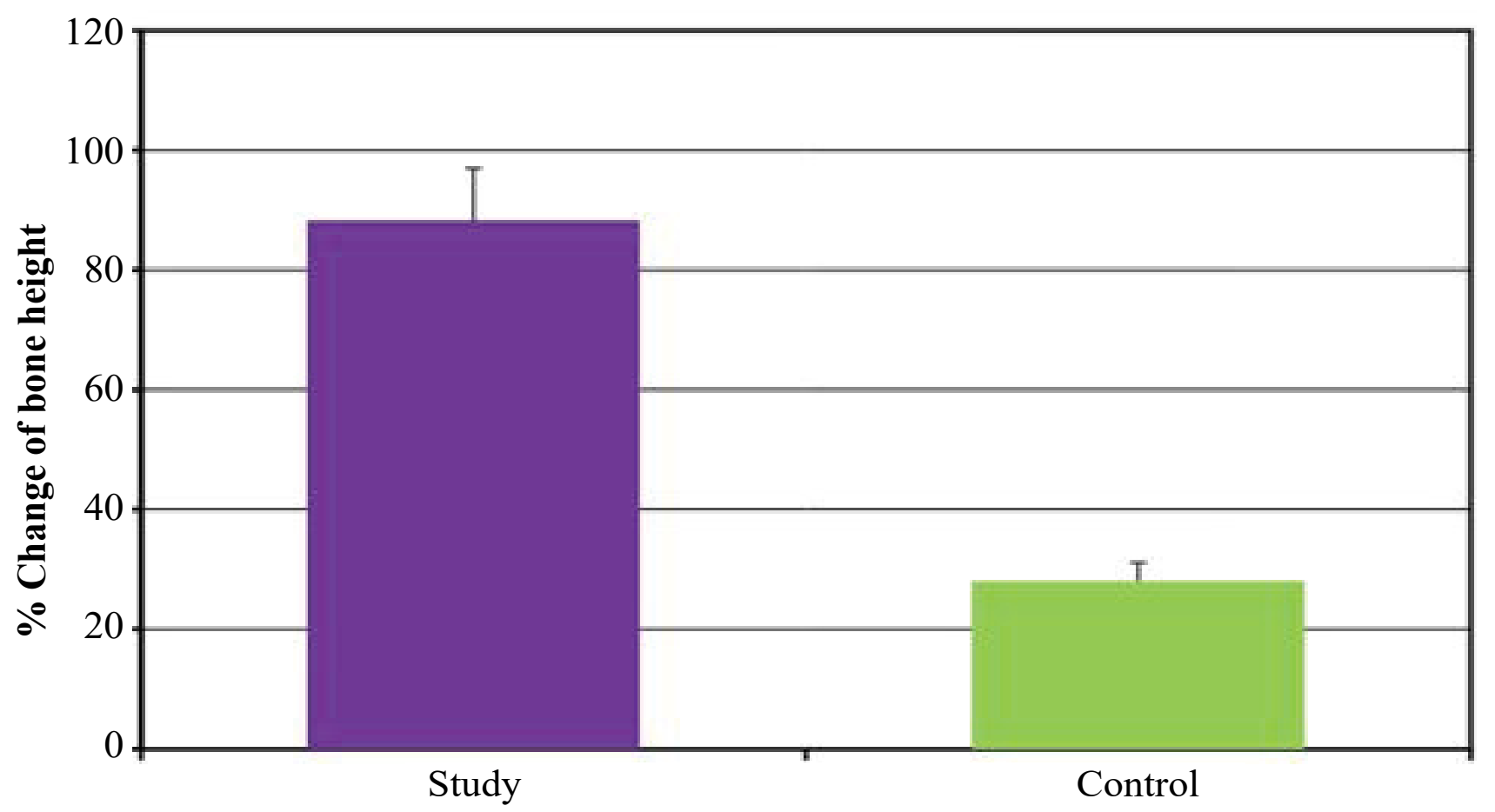

Figure 2: Bar chart showing changes in bone height from pre to postoperatively in both groups. 
difference, where $\left(P<0.002^{*}\right)$. The difference in mean value of bone density in between control and study groups pre and postoperatively was non-significant statistically Table 2 and Figure 3.

\section{Clinical postoperative outcomes}

All implants were inserted in molar region, twelve of them $(70.0 \%)$ were placed in the site of upper first molar and six $(30.0 \%)$ in the upper missed second molar site. There was no severing pain, hug swelling, suppuration or mobility detected during period of evaluation. Mild sinusitis was reported in two patients, it was subsided by third week postoperatively. There was no implant failure reported during period of evaluation. Postoperative assessment of the patient was as the following: Pain, swelling and implant stability.

A. Pain: The degree of pain was evaluated in all groups with the VAS scale. In the study group, the mean value of the pain was $6.25 \pm 0.89$ on the first day, 6.50 \pm 1.20 on the third day, $7.25 \pm 0.89$ on the fifth day, and $7.63 \pm 0.74$ on the seventh day. A statistically significant difference was detected in the mean value of the pain between the control and study groups from one to seven days. The pain was increased in the study more than in control with a statistically significant difference.

B. Swelling: Postoperative swelling was observed the second day after surgery and disappeared at tenday, no huge swelling was observed during the period of evaluation. The mean value of the swelling in the study group was $11.76 \pm 0.88$ on the first day, 11.68 \pm 1.02 on the third day, $12.64 \pm 0.96$ on the fifth day, and $12.08 \pm 0.96$ on the seventh day. The swelling was increased on the third, fifth, and seventh day with a significant statistical difference, where $\left(P \ll 0.001^{*}\right)$. In the control group, the mean value of the swelling was $12.51 \pm 1.14$ on the first day, $13.10 \pm 1.64$ on the third day, $12.80 \pm 1.34$ on the fifth day, and $12.52 \pm 1.13$ on the seventh day. The swelling increased at third, and decreased at fifth and seventh day with a non-significant statistical difference, where $(P<0.084)$. So, there was no statistically significant difference in the mean value of swelling between both groups from one to seven postoperative days.

C. Implant stability: The stability was evaluated in all groups with ostell. In the Study group, the mean value of the stability was $50.0 \pm 4.07$ pre-treatment and, $70.25 \pm$ 2.87 post-treatment. The stability was increased with a significant statistical difference, where $\left(P<<0.001^{*}\right)$. In the control group, the mean value of the stability was 58.25 \pm 1.67 pre-treatment and $71.63 \pm 2.13$ post-treatment. The stability was increased with a significant statistical difference, where $\left(P<0.001^{*}\right)$. There was no statistically significant difference in mean stability in the two groups.

\section{Discussion}

Rehabilitation of the atrophic maxilla with a dental implant constitutes a therapeutic problem, since bone augmentation is often required to enable placement, ensure the stability of a sufficient number and length of implants. Various grafting procedures and materials have been described. Maxillary sinus floor elevation is a highly predictable and reliable solution in many situations. However, the quest for the optimal protocol and the ideal grafting material to achieve high implant success rates, shorten treatment periods, and minimize morbidity is permanent and continuous [9].

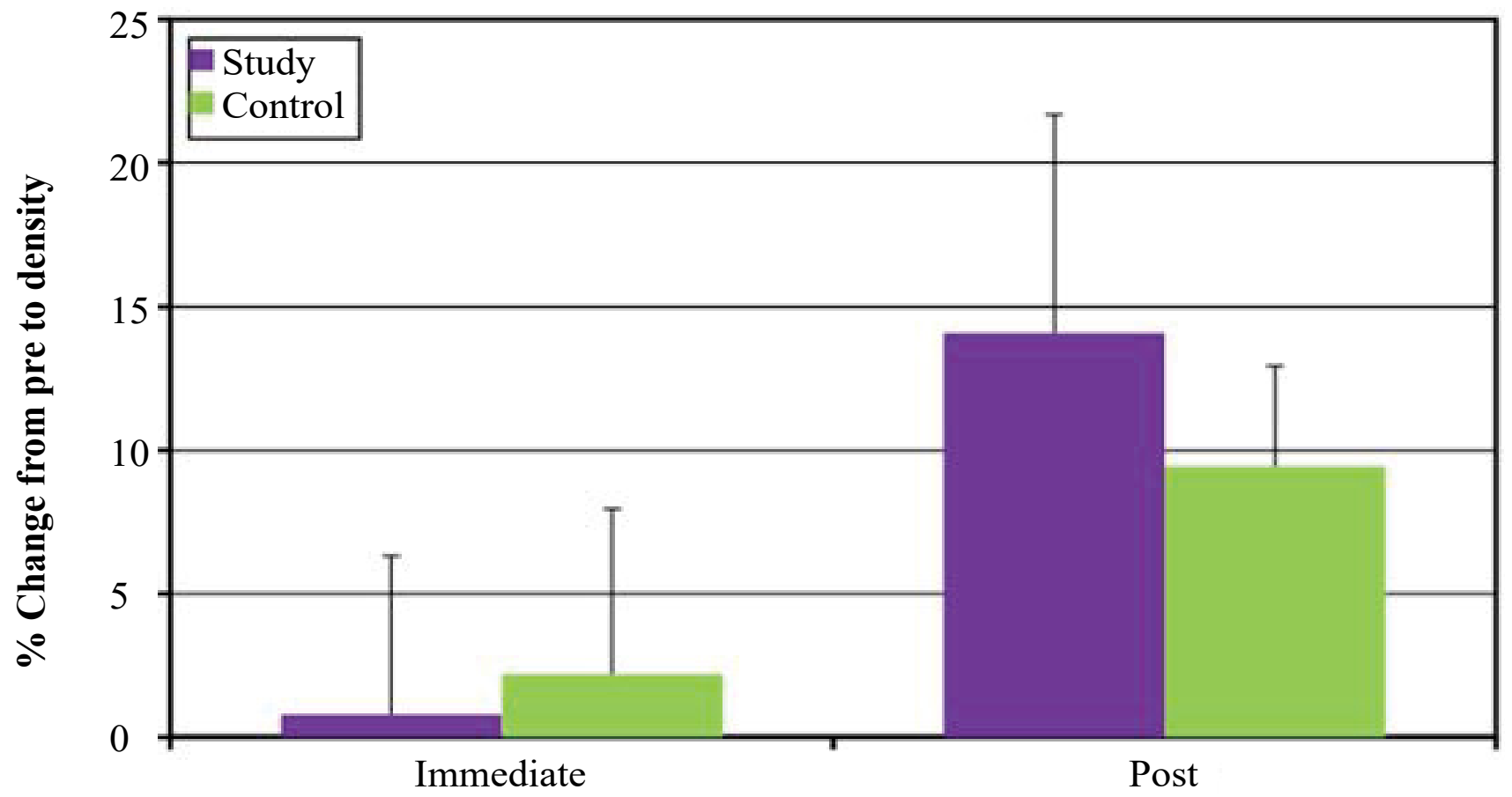

Figure 3: Bar chart showing changes of bone density in both groups. 
This study follows the one-stage procedure for dental implant placement associated with augmentation of the sinus floor in a severely atrophic maxilla, a minimum base of $5 \mathrm{~mm}$ for adequate implant stabilization and parallelism. Tarun [5], Inserted a dental implant in augmented sinus in a severely atrophic maxilla in a onestage procedure with a minimum base height of 4 to 5 $\mathrm{mm}$.

The residual bone height (RBH), measured from the crest of the ridge up to the sinus floor, for all patients, enrolled in this study was within the range of (5 to 9 $\mathrm{mm}$ ). The literature of Nkem [6] and Reiser [7], stated that the Transcrestal approach of osteotome to elevate sinus membrane can be performed with residual bone height beyond 4 to $5 \mathrm{~mm}$, also Davarpanah, et al. [8], proposed a modified osteotome technique, in which the bone thickness below the sinus was $\geq 5 \mathrm{~mm}$. A systematic review by Del Fabbro [10] suggested that transalveolar sinus augmentation with simultaneous implant placement could be a viable treatment option even in cases with minimal residual bone height, although the prognosis is still more favorable when the residual ridge is at least $5 \mathrm{~mm}$ high. On the other hand, Jensen OT, [11] recommended ( 7 to $9 \mathrm{~mm}$ ) at least for osteotome technique with simultaneous placement of implants. Furthermore, patients in RBH were $\leq 4 \mathrm{~mm}$ excluded as it is extremely difficult to gain primary stability in such cases.

In this study, care was taken to ensure high primary implant stability. This was achieved by using a thinner final drill $(3.5 \mathrm{~mm})$ than the usually used $(4 \mathrm{~mm})$ for $(4$ $\mathrm{mm}$ NeoBiotech IS-II active) root form with about $99 \%$ survival rates in the posterior maxilla with poor bone [12].

In this context, it was decided to conduct this study to assess whether the oxidized regenerated cellulose graft will show promising results in augmentation and bone gain around the implants and to be a safe alternative to other bone graft materials.

Sixteen maxillary sinuses were enrolled in this study. They were divided randomly into two groups, study group, in which ORC grafts were used in trans alveolar sinus lifting and implant installation, and control group, in which no grafts were used. This sample size is approximate to that of Hussein and Hassan [13], to our knowledge, not many previous studies made such a comparison between these groups. To limit the variables that may affect the results of the current study, certain inclusion and exclusion criteria were set for patient selection. Normal healthy patients were selected. Smoking was considered a contraindication, as patients were informed that it can reduce the success rates of the procedure and compromise the sinus lift procedure. Baig MR [14] even stated that the failure rate of implants placed in grafted maxillary sinuses of smokers is more than twice that seen in non-smokers. Those who were suffering from a pre-existing sinus disease were excluded because many studies have established the direct relationship between the increased incidence of implant complications and the presence of sinus pathology [15].

The clinical evaluation of this study showed (VAS) range for pain was mild to moderate for the study and mild for the control group. The pain was absent in all patients after $1^{\text {st }}$ week at the $3^{\text {rd }}, 6^{\text {th }}$, and $12^{\text {th }}$ weeks of observation. On comparing both groups, the pain was found to be absent after $1^{\text {st }}$ week and a significant reduction of pain was noticed with time. On the $1^{\text {st }}$ day, the pain was higher in both groups because of soft tissue elevation, drilling of bone, pressure effect of implant insertion, bone cutting, and sinus membrane elevation. Similar findings were observed by Kent and Block, [16] who evaluated clinical outcomes of dental implant placement and sinus floor elevation and observed that there was no significant pain after sinus lift surgery postoperatively. Wiltfang, et al. [17] observed pain reduction after sinus lift surgery with time but found 2 patients with sinusitis-related pain which they found to be due to migration of cancellous bone sequestra into maxillary sinus for which they performed minus copy and removal of sequestrum. At day 1-7 there was a statistically non-significant difference in mean swelling in the two groups. There was no implantitis or infection or sinusitis, the ORC graft showed a high increase in bone height and implant primary stability was measured immediately and 6 months postoperative. Radiographically, immediate and 6 months postoperative CBCT was done for regular follow up and evaluate density of neo formed bone.

According to the Osseo-integration definition as described by Branemark [18], a direct structural and functional connection between ordered, living bone and the surface of a load-carrying implant under light microscopic level. The gold standard method used to evaluate the degree of osseointegration was the microscopic or the histologic analysis. Despite this fact, these methods are considered to be invasive and contraindicating ethical issues in the case of clinical human studies. Therefore, this study depended on the measurement of implant stability using resonance frequency analysis (RFA).

The measurements of implant stability using Osstell were obtained immediately after implant insertion, to assess the primary stability and set a baseline for comparison, and after 6 months to assess the secondary stability before implant loading. The mean value of ISQ immediately in Group A and Group B was $50 \pm 4.07$ and $70.25 \pm 2.87$ respectively. While the mean value of ISQ 6 months postoperatively in Group A and Group B was $58.25 \pm 1.67$ and $71.63 \pm 2.13$ respectively, where the secondary stability reported in this study was in correspond to what reported by Kolerman [19] And 
the mean of change in the ISQ value readings between immediate and 6 months postoperatively in Group A and Group B was $19.9 \pm 5.16$ and $13 \pm 4$ respectively.

The radiographic parameters of this study include measuring the bone height and density in the study and control groups, where bone height in the study group was $5.93 \pm 0.79$ pre-treatment and, $11.13 \pm 1.36$ posttreatment and in control group, was $8.60 \pm 0.21$ pretreatment and, $11.0 \pm 0.0$ post-treatment. The Bone Height was increased significantly in the study group than the control. The results of the study group agree with the results of Hussein and Hassan [20] which was 11,36 for the study group and agree with the result of Yan [21] which showed no significant difference in the survival rate between the non-graft group and the graft group (RR: $1.02 ; p=0.18$ ). No statistically significant difference in marginal bone loss was detected between the groups at 12 months $(0.57, p=0.07)$ or 36 months $(0.05, p=0.61)$. The endo-sinus bone gain in the nongraft group was significantly lower than in the graft group at 12 months $(-1.10, p=0.0001)$ and 36 months $(-0.74, p=0.02)$. Al's bone height achieved with a less invasive transrectal approach was nearly comparable to the results reported by other clinical studies using autogenous bone, osteon II [22,23]. However, the Higher bone gain achieved by other clinical studies is due to the immediate postoperative measurement of bone height without accounting for bone graft resorption during the healing period $[24,25]$.

The mean total neoformed bone density for both groups was $490.45 \mathrm{HU}$ that lies within the D3 category bone type which presented with $70 \%$ of sinusal dental implants. Regarding both groups, the mean density of the study and control groups reached 456.4 and 524.5 $\mathrm{HU}$, respectively, with a statistically non-significant difference in mean density in the two groups. Another study reported asignificant increase in density of bone formed after augmented sinus lifting with Nanobone more than that of newly formed bone after nonaugmented sinus [26].

Lee, et al. [27] evaluated bone graft density of augmented sinus by CBCT after 20 weeks, and reporting $312 \mathrm{HU}$ as a mean, which became $512.75 \mathrm{HU}$ after 1 year, the authors concluded that there was a direct proportion between progressive maturation of bone mineral density assessed by $\mathrm{HU}$ and amount of new bone assessed histologically.

The result of this study concluded that ORC was a feasible substitute to other grafting materials as technically safe, cheap, fast and its application does not require the sophisticated procedure. When it becomes saturated with blood it swells into a black gelatinous mass to provide tenting effect along with installed dental implant maintaining elevated Schneider membrane against collapse, this space is then populated by boneforming cells to create new bone which is acceptable clinically as evidenced by implant immobility (acceptable secondary stability) and radiographically crosses $11 \mathrm{~mm}$ of minimum augmentation height. These results agree with the clinical studies of Fugazzotto and Vlassis [28] and Kim, et al. [29].

\section{Conclusion}

According to the results of this study, we could conclude that:

- Osteotome mediated maxillary sinus lifting with simultaneous implant placement using oxidized regenerated cellulose graft is a promising technique compared to other grafting materials as technically safe, cheap, fast and its application does not require a sophisticated procedure through trans alveolar sinus membrane elevation and simultaneous implant insertion.

- Oxidized regenerated cellulose could be considered as an alternative reasonable grafting material with comparable outcomes to osteon II with less postoperative complications.

- If the residual bone below sinus floor $\geq 8 \mathrm{~mm}$, it's not required to use graft materials for sinus floor augmentation.

- If the residual bone height is $\leq 6 \mathrm{~mm}$ ORC could be used as filling material for crestal sinus floor augmentation.

\section{References}

1. Salah G, Mohammed G, Ramy B (2020) Bone Formation After Maxillary Sinus Floor Elevation Using Titanium Mesh Versus Titanium Screws Without Any Graft Material: A Preliminary Study. Advanced Dental Journal 2: 201-208.

2. Shalu C, Ramesh R, Komal M (2018) Maxillary Sinus Augmentation. J Indian Soc Periodontol 22: 468-473.

3. Sanath S, Naushad P, Vidya B, Shenoy KK (2014) Short Implants: A New Dimension In Rehabilitation Of Atrophic Maxilla And Mandible. Int J Oral Maxillofac Implants 4: 6670.

4. Renouard F, Nisand D (2005) Short implants in severely resorbed maxilla: A 2-Year retrospective clinical study. Clin Implant Dent Rel Res 7: 104.

5. Tarun K, UllasA (2015) Maxillary Sinus Augmentation. Journal of the International Clinical Dental Research Organization 7: 81-93.

6. Nkem O (2019) Osteotome technique: A minimally invasive way to increase bone for dental implant placement in the posterior maxilla and prevent sinus membrane perforation for single and multiple teeth replacements. J Med Clin Res Rev 3: 1-6.

7. Reiser G, Rabinovitz Z, Bruno J, Damoulis PD, Griggin TJ (2001) Evaluation of maxillary sinus membrane response following elevation with the crestal osteotome technique in human cadavers. Int J Oral Maxillofacial Implant 16: 833840.

8. Davarpanah, Henry M, Hage G, Lazzara R (2001) Modified Osteotome Technique. Int J Periodontics Restorative Dent 21: 599-607. 
9. Gökhan G, Yasar Ö (2017) Book paranasal sinuses chapter 2 maxillary sinus augmentation for dental implants. Intech Open Science 1: 39-54.

10. Del MF, Corbella S, Weinstein T, Ceresoli V, Taschieri S (2012) Implant Survival Rates After Osteotome-Mediated Maxillary Sinus Augmentation: A Systematic Review. Clin Implant Dent Relat Res 14: e159-e168.

11. Jensen OT, Shulman LB, Block MS, Lacono VJ (1998) Report of the sinus consensus conference of 1996. Int J Oral Maxillofac Implants 13: 11-45.

12. Stricker A, Voss $P$, Gutwald R, Schramm A, Schmelzeisen $R$ (2003) Maxillary sinus floor augmentation with autogenous bone grafts to enable placement of sla-surfaced implants: preliminary results after $15-40$ months. Clinical Oral Implants Research 14: 207-212.

13. Rodrigo A, José V, Mariano H, Bullon $P$, Farhall JF, et al. (2021) Sinus floor elevation via an osteotome technique without biomaterials. Int J Environ Res Public Health 18: $1-13$.

14. Baig M, Rajan M (2007) Effects of smoking on the outcome of implant treatment: a literature review. Indian Journal of Dental Research 18: 190-195.

15. Kan J, Rungcharassaeng K, Lozada J, Goodacre CJ (1999) Effects of smoking on implant success in grafted maxillary sinuses. The Journal Of Prosthetic Dentistry 82: 307-311.

16. Luis C, Javier R, David P (2015) Influence of smoking and oral hygiene on success of implants placed after direct sinus lift. Journal of Oral Science \& Rehabilitation 1: 70-75.

17. Wiltfang J, Schultze-Mosgau S, Merten $H$, Kessler $P$, Ludwing A, et al. (2000) Endoscopic and ultrasonographic evaluation of the maxillary sinus aft er combined sinus floor augmentation and implant insertion. Oral Surg Oral Med Oral Pathol Oral Radiol Endod 89: 288-291.

18. Branemark $P$ (1983) Osseointegration and its experimental studies. Journal Of Prosthetic Dentistry 50: 399-410.

19. Kolerman R, Moses O, Artzi Z, Barnea E, Tal H (2011) Maxillary sinus augmentation by the crestal core elevation technique. J Periodontol 82: 41-51.
20. Oto A, Remer E, O'malley C, Tkach JA, Gill IS (1999) Mr Characteristics Of Oxidized Cellulose (Surgicel). Ajr Am J Roentgenol 172: 1481-1484.

21. Yan M, Liu R, Bai S, Wang M, Xia H, et al. (2018) Transalveolar sinus floor lift without bone grafting in atrophic maxilla: A meta-analysis. Scientific Reports 8: 1451.

22. Rao G, Reddy S (2014) Antral balloon sinus elevation and grafting prior to dental implant placement: Review of 34 cases. Int J Oral Maxillofac Implants 29: 414-418.

23. Asmael H, Lateef $T$ (2016) An assessment of the efficacy of sinus balloon technique on transcrestal maxillary sinus floor elevation surgery. J Bagh Coll Dent 28: 109-113.

24. Amr KA, Walid AG, Abdelbadeeabdallah MA (2020) Comparative study between two different bone grafts for enhancing osteo-integration of immediate implant. Official Dental Journal Of Suez Canal University Dental Science 1: $113-120$

25. Nakajima K, Kusama $Y$ (2016) Cone beam computed tomography evaluation of bone remodeling following the osteotome sinus floor elevation technique for future site development. Int J Periodontics Restorative Dent 36: 497505.

26. Shawky K, Shehab M, Hassanein F (2019) Density of bone formed after sinus augmentation with nanobone versus that of bone formed after sinus lift with tenting technique: Preliminary study. Egyptian Journal of Oral and Maxillofacial Surgery 10: 1-8.

27. Lee C, Prasad H, Suzuki J, Stover JD, Rohrer MD (2011) The correlation of bone mineral density and histologic data in the early grafted maxillary sinus: a preliminary report. Implant Dent 20: 202-214.

28. Fugazzotto $P$, Vlassis J (1998) Long-term success of sinus augmentation using various surgical approaches and grafting materials. Int J Oralmaxillofac Implants 13: 52-58.

29. Kim Y, Choe G, Yun P (2008) Management of perforated sinus membrane using absorbable haemostat and fibrin adhesive for sinus lift procedure. Asian $\mathrm{J}$ Oral Maxillofac Surg 20: 129-134. 